\title{
Some Properties of $\boldsymbol{k}$-Jacobsthal Numbers with Arithmetic Indexes
}

\author{
Deepika Jhala $^{1, *}$, G.P.S. Rathore ${ }^{2}$, Kiran Sisodiya ${ }^{1}$ \\ ${ }^{1}$ School of Studies in Mathematics, Vikram University, Ujjain (India) \\ ${ }^{2}$ College of Horticulture, Mandsaur (M.P.) \\ *Corresponding author: jhala.deepika28@gmail.com
}

Received June 08, 2014; Revised July 13, 2014; Accepted July 23, 2014

\begin{abstract}
In this paper, we derive various formulae for the sum of k-Jacobsthal numbers with indexes in an arithmetic sequence, say $a n+r$ for fixed integers $a$ and $r$. Also, we describe generating function and the alternated sum formula for k-Jacobsthal numbers with indexes in an arithmetic sequence.
\end{abstract}

Keywords: $k$-jacobsthal numbers, binet formula, generating function

Cite This Article: Deepika Jhala, G.P.S. Rathore, and Kiran Sisodiya, "Some Properties of $k$-Jacobsthal Numbers with Arithmetic Indexes." Turkish Journal of Analysis and Number Theory, vol. 2, no. 4 (2014): 119124. doi: 10.12691/tjant-2-4-3.

\section{Introduction}

The Fibonacci sequence and the Lucas sequence are the two shining stars in the vast array of integer sequences. They have fascinated both amateurs and professional mathematicians for centuries. Also they continue to charm us with their beauty, their abundant applications, their ubiquitous habit of occurring in totally surprising and unrelated places. Fibonacci numbers have been generalized by different authors. Some authors have generalized the Fibonacci sequence by preserving the recurrence relation and altering the first two terms of the sequence $[10,12,17]$, while others have generalized the Fibonacci sequence by preserving the first two terms but altering the recurrence relation slightly $[14,15,18]$.

More recently, Fibonacci, Lucas, Pell, Pell-Lucas, Modified Pell, Jacobhstal, Jacobsthal-Lucas sequences were generalized for any positive real number $k$. Also the study of the $k$-Fibonacci sequence, the $k$-Lucas sequence, the $k$-Pell sequence, the $k$-Pell-Lucas sequence, the Modified $k$-Pell sequence, the $k$-Jacobhstal sequence and the $k$-Jacobsthal Lucas sequence appeared (see $[1-6,8,13]$ ). Falcon and plaza [7] has given several formulae for the sum of $k$-Fibonacci numbers with indexes in an arithmetic sequence. Falcon [9] defines the $k$-Lucas number with indexes in an arithmetic sequence. Also, deduced generating function and several sum formulae for these numbers with indexes in an arithmetic sequence.

\section{1. $k$ - Jacobsthal and $k$ - Jacobsthal Lucas Numbers}

\subsubsection{Definition 1.1 ( $k$-Jacobsthal Numbers)}

For any positive real number $k$, the $k$-Jacobsthal sequence say [13] is defined recurrently by

$$
J_{k, n+1}=k J_{k, n}+2 J_{k, n-1} ; n \geq 1
$$

with initial conditions $J_{k, 0}=0, J_{k, 1}=1$.

Particular cases of definition (1.1)

If $k=1$, we obtain the Jacobsthal sequence

$\{0,1,1,3,5,11,21 \ldots\}(\mathrm{A} 001045)[16]$

If $k=2$ we obtain the sequence $\{0,1,2,6,16,44,120, \ldots\}$ A002605 [16].

First few terms of the k-Jacobsthal numbers (1.1) are

$$
\begin{aligned}
& J_{k, 0}=0, J_{k, 1}=1, J_{k, 2}=k, J_{k, 3}=k^{2}+2, \\
& J_{k, 4}=k^{3}+4 k, J_{k, 5}=k^{4}+6 k^{2}+4, \\
& J_{k, 6}=k^{5}+8 k^{3}+12 k
\end{aligned}
$$

Some of the interesting properties that the k-Jacobsthal sequence satisfies are summarized as below [13]:

\subsubsection{Binet's Formula}

The Binet formula for the $n^{\text {th }} \mathrm{k}$-Jacobsthal numbers is

$$
J_{k, n}=\frac{\lambda_{1}^{n}-\lambda_{2}^{n}}{\lambda_{1}-\lambda_{2}}
$$

where $\lambda_{1}=\frac{k+\sqrt{k^{2}+8}}{2}, \lambda_{2}=\frac{k-\sqrt{k^{2}+8}}{2}$ are the roots of the characteristic equation $r^{2}=k r+2$ associated to the recurrence relation defined in equation (1.1) and $\lambda_{1}>\lambda_{2}, \lambda_{1}+\lambda_{2}=k, \lambda_{1} \lambda_{2}=-2, \lambda_{1}-\lambda_{2}=\sqrt{k^{2}+8}$.

\subsubsection{Catalan's Identity}

$$
J_{k, n-r} J_{k, n+r}-J_{k, n}^{2}=(-1)^{n+1-r} J_{k, r}^{2} 2^{n-r}
$$

\subsubsection{D'Ocagne Identity}

If $m>n$ then 


$$
J_{k, m} J_{k, n+1}-J_{k, m+1} J_{k, n}=(-2)^{n} J_{k, m-n}
$$

\subsubsection{Convolution Product}

$$
J_{k, n+m}=J_{k, n+1} J_{k, m}+2 J_{k, n} J_{k, m-1}
$$

Now, we introduce the $k$-Jacobsthal Lucas sequence, whose recurrence relation is the same as the $k$-Jacobsthal sequence.

\subsection{Definition 1.2}

For any positive real number $k$, the $k$-Jacobsthal-Lucas sequence say [2] is defined recurrently by

$$
j_{k, n+1}=k j_{k, n}+2 j_{k, n-1} ; \text { for } n \geq 1
$$

with initial conditions $j_{k, 0}=2, \quad j_{k, 1}=k$.

As particular cases:

If $k=1$, we obtain the Jacobsthal-Lucas sequence [11] $\{2,1,5,7,17,31,65, \ldots\}$ (A014551) [16].

If $k=2$, we obtain the sequence $\{2,2,6,14,34,82,198, \ldots\}$ A080040 [16].

First few terms of the $k$-Jacobsthal Lucas numbers equation (1.6) are

$$
\begin{aligned}
& j_{k, 0}=2, j_{k, 1}=k, j_{k, 2}=k^{2}+4, j_{k, 3}=k^{3}+6 k \\
& j_{k, 4}=k^{4}+8 k^{2}+8, j_{k, 5}=k^{5}+10 k^{3}+20 k \\
& j_{k, 6}=k^{6}+12 k^{4}+36 k^{2}+16
\end{aligned}
$$

Some of the interesting properties that the $k$-Jacobsthal Lucas sequence satisfies are summarized as below [2].

\subsubsection{Binet's Formula}

The Binet formula for the $k$-Jacobsthal numbers is

$$
j_{k, n}=\lambda_{1}^{n}+\lambda_{2}^{n},
$$

where $\lambda_{1}=\frac{k+\sqrt{k^{2}+8}}{2}, \lambda_{2}=\frac{k-\sqrt{k^{2}+8}}{2}$ are the roots of the characteristic equation $r^{2}=k r+2, \lambda_{1}>\lambda_{2}$ $\lambda_{1}+\lambda_{2}=k, \lambda_{1} \lambda_{2}=-2$ and $\lambda_{1}-\lambda_{2}=\sqrt{k^{2}+8}$ associated to the recurrence relation defined in equation (1.6).

\subsubsection{Catalan's Identity}

$$
j_{k, n-r} j_{k, n+r}-j_{k, n}^{2}=(-2)^{n-r}\left(j_{k, r}^{2}-4(-2)^{r}\right)
$$

\subsubsection{D'Ocagne Identity}

If $\mathrm{m}>\mathrm{n}$ then

$$
\begin{aligned}
& j_{k, m} j_{k, n+1}-j_{k, m+1} j_{k, n} \\
& =(-2)^{n} \sqrt{k^{2}+8}\left[j_{k, m-n}-2\left(\frac{k+\sqrt{k^{2}+8}}{2}\right)^{m-n}\right]
\end{aligned}
$$

Now, we prove some properties of the k-Jacobsthal numbers that we will be needed later.

$$
J_{k, m+n}=J_{k, n+1} J_{k, m}+2 J_{k, n} J_{k, m-1}
$$

Proof: Taking R.H.S. and applying the Binet's formula for k-Jacobsthal numbers

$$
\begin{aligned}
& J_{k, n+1} J_{k, m}+2 J_{k, n} J_{k, m-1} \\
& =\left(\frac{\lambda_{1}^{n+1}-\lambda_{2}^{n+1}}{\lambda_{1}-\lambda_{2}}\right)\left(\frac{\lambda_{1}^{m}-\lambda_{2}^{m}}{\lambda_{1}-\lambda_{2}}\right) \\
& +2\left(\frac{\lambda_{1}^{n}-\lambda_{2}^{n}}{\lambda_{1}-\lambda_{2}}\right)\left(\frac{\lambda_{1}^{m-1}-\lambda_{2}^{m-1}}{\lambda_{1}-\lambda_{2}}\right) \\
& =\frac{1}{\left(\lambda_{1}-\lambda_{2}\right)^{2}}\left\{\begin{array}{l}
\left(\lambda_{1}^{n+1}-\lambda_{2}^{n+1}\right)\left(\lambda_{1}^{m}-\lambda_{2}^{m}\right) \\
+2\left(\lambda_{1}^{n}-\lambda_{2}^{n}\right)\left(\lambda_{1}^{m-1}-\lambda_{2}^{m-1}\right)
\end{array}\right\} \\
& =\frac{1}{\left(\lambda_{1}-\lambda_{2}\right)^{2}}\left\{\begin{array}{l}
\lambda_{1}^{m+n}\left(\lambda_{1}-\lambda_{2}\right) \\
-\lambda_{2}^{m+n}\left(\lambda_{1}-\lambda_{2}\right)
\end{array}\right\} \\
& =\left(\frac{\lambda_{1}^{m+n}-\lambda_{2}^{m+n}}{\lambda_{1}-\lambda_{2}}\right)=J_{k, m+n}[\text { From equation (1.2) }]
\end{aligned}
$$

That is $J_{k, m+n}=J_{k, n+1} J_{k, m}+2 J_{k, n} J_{k, m-1}$

$$
J_{k, m} J_{k, n+1}-J_{k, m+1} J_{k, n}=(-2)^{n} J_{k, m-n}
$$

Proof: We will prove this by using the Mathematical induction method. For

$$
\begin{aligned}
& n=0, \\
& J_{k, m} J_{k, n+1}-J_{k, m+1} J_{k, n} \\
& =J_{k, m} J_{k, 1}-J_{k, m+1} J_{k, 0} \\
& =J_{k, m} \cdot 1-J_{k, m+1} \cdot 0=J_{k, m}
\end{aligned}
$$

we see that it is true for $n=0$.

Now for $n=1$, we have

$$
\begin{aligned}
& J_{k, m} J_{k, 2}-J_{k, m+1} J_{k, 1}=J_{k, m} \cdot k-J_{k, m+1} \cdot 1 \\
& \text { [From equation (1.1)] } \\
& =J_{k, m+1}-2 J_{k, m-1}-J_{k, m+1}=-2 J_{k, m-1}
\end{aligned}
$$

again we see that it is true for $n=1$.

Now, suppose the formula is true until $(n-1)$ :

$$
\left.\begin{array}{l}
J_{k, m} J_{k, n-1}-J_{k, m+1} J_{k, n-2}=(-2)^{n-2} J_{k, m-(n-2)} \\
J_{k, m} J_{k, n}-J_{k, m+1} J_{k, n-1}=(-2)^{n-1} J_{k, m-(n-1)}
\end{array}\right\}
$$

Then,

$$
\begin{aligned}
& J_{k, m} J_{k, n+1}-J_{k, m+1} J_{k, n} \\
& =J_{k, m}\left(k J_{k, n}+2 J_{k, n-1}\right)-J_{k, m+1}\left(k J_{k, n-1}+2 J_{k, n-2}\right) \\
& {[\text { From equation }(1.1)]} \\
& =k\left(\mathrm{~J}_{k, m} L_{k, n}-J_{k, m+1} J_{k, n-1}\right) \\
& +2\left(\mathrm{~J}_{k, m} J_{k, n-1}-J_{k, m+1} J_{k, n-2}\right) \\
& =(-2)^{n-1}\left(k J_{k, m-(n-1)}-J_{k, m-(n-2)}\right) \\
& =(-2)^{n} J_{k, m-n}
\end{aligned}
$$

That is $J_{k, m} J_{k, n+1}-J_{k, m+1} J_{k, n}=(-2)^{n} J_{k, m-n}$. 
2. On the $k$-Jacobsthal Numbers of Kind $a n+r$

In this section, we shall derive some formulae for the sums of the k- Jacobsthal numbers with index in an arithmetic sequence, say $a n+r$ for fixed integer $a$ and $r$ such that $0 \leq r \leq a-1$. Also, we have discuss generating function for these numbers with index in an arithmetic sequence.

First we prove following lemmas that will be needed later.

\subsection{Lemma 2.1}

For all integers $n \geq 1$,

$$
\lambda_{1}^{n}+\lambda_{2}^{n}=J_{k, n+1}+2 J_{k, n-1}
$$

Proof: Taking R.H.S. and applying the Binet's formula for k-Jacobsthal numbers and $\lambda_{1} \cdot \lambda_{2}=-2$.

$$
\begin{aligned}
& J_{k, n+1}+2 J_{k, n-1} \\
& =\frac{1}{\lambda_{1}-\lambda_{2}}\left(\lambda_{1}^{n+1}-\lambda_{2}^{n+1}+2 \lambda_{1}^{n-1}-2 \lambda_{2}^{n-1}\right) \\
& =\lambda_{1}^{n}+\lambda_{2}^{n}
\end{aligned}
$$

That is $J_{k, n+1}+2 J_{k, n-1}=\lambda_{1}^{n}+\lambda_{2}^{n}$

\subsection{Lemma 2.2}

$$
\begin{aligned}
& J_{k, a(n+2)+r}=\left(2 J_{k, a-1}+J_{k, a+1}\right) J_{k, a(n+1)+r} \\
& -(-2)^{a} J_{k, a n+r}
\end{aligned}
$$

Proof: Taking R.H.S. and applying Binet's formula and Lemma 2.1.

$$
\begin{aligned}
& \left(2 J_{k, a-1}+J_{k, a+1}\right) J_{k, a(n+1)+r}-(-2)^{a} J_{k, a n+r} \\
& =\left(\lambda_{1}^{a}+\lambda_{2}^{a}\right)\left(\frac{\lambda_{1}^{a(n+1)+r}-\lambda_{2}^{a(n+1)+r}}{\lambda_{1}-\lambda_{2}}\right)-(-2)^{a} J_{k, a n+r} \\
& =\left(\frac{\lambda_{1}^{a(n+2)+r}-\lambda_{2}^{a(n+2)+r}}{\lambda_{1}-\lambda_{2}}\right) \\
& +(-2)^{a}\left(\frac{\lambda_{1}^{a n+r}-\lambda_{2}^{a n+r}}{\lambda_{1}-\lambda_{2}}\right)-(-2)^{a} J_{k, a n+r}=J_{k, a(n+2)+r}
\end{aligned}
$$

now, since $j_{k, n}=J_{k, n+1}+2 J_{k, n-1}$, then the above formula can be rewritten as

$$
J_{k, a(n+2)+r}=j_{k, \mathrm{a}} J_{k, a(n+1)+r}-(-2)^{a} J_{k, a n+r}
$$

equation (2.2) gives the general term of the k-Jacobsthal sequence $\left\{J_{k, a n+r}\right\}_{n=0}^{\infty}$ as a linear combination of the two preceding terms.

2.2.1. Generating Function for the Sequence $\left\{J_{k, a n+r}\right\}_{n=0}^{\infty}$
Suppose that $\mathfrak{I}_{a, r}(k, x)$ be the generating function of the sequence $\left\{J_{k, a n+r}\right\}$ with $0 \leq r \leq a-1$. That is

$$
\begin{aligned}
& \mathfrak{J}_{a, r}(k, x)=J_{k, r}+J_{k, a+r} x \\
& +J_{k, 2 a+r} x^{2}+J_{k, 3 a+r} x^{3}+\ldots
\end{aligned}
$$

Now multiplying both sides by the algebraic expression $\left(1-j_{k, a} x+(-2)^{a} x^{2}\right)$, we get

$$
\begin{aligned}
& \left(1-j_{k, a} x+(-2)^{a} x^{2}\right) \mathfrak{J}_{a, r}(k, x)=\left(1-j_{k, a} x+(-2)^{a} x^{2}\right), \\
& \left(J_{k, r}+J_{k, a+r} x+J_{k, 2 a+r} x^{2}+J_{k, 3 a+r} x^{3}+\ldots\right) \\
& =J_{k, r}+\left(J_{k, a+r}-j_{k, a} J_{k, r}\right) x \\
& +\left(J_{k, 2 a+r}-j_{k, a} J_{k, a+r}+(-2)^{a} J_{k, r}\right) x^{2}+\ldots \\
& =J_{k, r}+\left(J_{k, a+r}-j_{k, a} J_{k, r}\right) x \\
& +\sum_{n \geq 2}\left(\begin{array}{l}
J_{k, a(n+2)+r}-j_{k, a} J_{k, a}(n+1)+r \\
+(-2)^{a} J_{k, a n+r}
\end{array}\right) x^{n}
\end{aligned}
$$

Now, from equation (2.2), the summation of right hand side of the above equation vanishes. That is

$$
\begin{aligned}
& \left(1-j_{k, a} x+(-2)^{a} x^{2}\right) \mathfrak{J}_{a, r}(k, x) \\
& =J_{k, r}+\left(J_{k, a+r}-j_{k, a} J_{k, r}\right) x
\end{aligned}
$$

from equation (1.10), we have

$$
\begin{aligned}
& J_{k, a+r}=J_{k, r+1} J_{k, a}+2 J_{k, r} J_{k, a-1} \\
& J_{k, a+r}-J_{k, r} j_{k, a}=J_{k, r+1} J_{k, a}-J_{k, r} J_{k, a+1} \\
& J_{k, a+r}-J_{k, r} j_{k, a}=(-2)^{r} J_{k, \mathrm{a}-r}[\text { From (1.11)] }
\end{aligned}
$$

Hence equation (2.4) becomes

$$
\begin{aligned}
& \left(1-j_{k, a} x+(-2)^{a} x^{2}\right) \mathfrak{J}_{a, r}(k, x) \\
& =J_{k, r}+(-2)^{r} J_{k, \mathrm{a}-r} x \\
& \mathfrak{J}_{\mathrm{a}, \mathrm{r}}(k, x)=\frac{J_{k, \mathrm{r}}+(-2)^{r} J_{k, \mathrm{a}-\mathrm{r}} x}{\left(1-j_{k, \mathrm{a}} x+(-2)^{a} x^{2}\right)}
\end{aligned}
$$

\section{Particular cases:}

For the different values of $a$ and $r$, the generating function of the sequences $\left\{J_{k, a n+r}\right\}$ are:

1) If $a=1$ and then $r=0$,

$$
\mathfrak{I}_{1,0}(k, x)=\frac{J_{k, 0}+J_{k, 1} x}{\left(1-j_{k, 1} x+(-2) x^{2}\right)}=\frac{x}{1-k x-2 x^{2}}
$$

which is the generating function of the $k$-Jacobsthal sequence [13].

2) If $a=2$, then

At $r=0$,

$\Im_{2,0}(k, x)=\frac{J_{k, 0}+(-2)^{0} J_{k, 2} x}{1-j_{k, 2} x+(-2)^{2} x^{2}}=\frac{k x}{1-\left(k^{2}+4\right) x+4 x^{2}}$

At $r=1$ 
$\mathfrak{I}_{2,1}(k, x)=\frac{J_{k, 1}+(-2)^{1} J_{k, 1} x}{1-j_{k, 2} x+(-2)^{2} x^{2}}=\frac{1-2 x}{1-\left(k^{2}+4\right) x+4 x^{2}}$

3) If $a=3$, then

At $r=0$,

$\mathfrak{I}_{3,0}(k, x)=\frac{J_{k, 0}+(-2)^{0} J_{k, 3} x}{1-j_{k, 3} x+(-2)^{3} x^{2}}=\frac{\left(k^{2}+2\right) x}{1-\left(k^{3}+6 k\right) x-8 x^{2}}$

At $r=1$,

$\mathfrak{J}_{3,1}(k, x)=\frac{J_{k, 1}+(-2)^{1} J_{k, 2} x}{1-j_{k, 3} x+(-2)^{3} x^{2}}=\frac{1-2 k x}{1-\left(k^{3}+6 k\right) x-8 x^{2}}$

At $r=2$,

$\mathfrak{I}_{3,2}(k, x)=\frac{J_{k, 2}+(-2)^{2} J_{k, 1} x}{1-j_{k, 3} x+(-2)^{3} x^{2}}=\frac{k+4 x}{1-\left(k^{3}+6 k\right) x-8 x^{2}}$

2.2.2. Sum of $\boldsymbol{k}$-Jacobsthal numbers with arithmetic index $a n+r$

In this section, we have discuss the sum formula for the $k$-Jacobsthal numbers with arithmetic index $a n+r$, where $a$ and $r$ are fixed integer such that $0 \leq r \leq a-1$.

\subsection{Theorem 2.3}

Sum of k-Jacobsthal number of kind $a n+r$ is

$$
\begin{array}{r}
J_{k, a(n+1)+r}-(-2)^{a} J_{k, a n+r} \\
\sum_{i=0}^{n} J_{k, a i+r}=\frac{-J_{k, r}-(-2)^{r} J_{k, a-r}}{J_{k, a+1}+2 J_{k, a-1}-(-2)^{a}-1}
\end{array}
$$

Proof: Applying Binet's formula for the k- Jacobsthal numbers, we have

$$
\begin{aligned}
& \sum_{i=0}^{n} J_{k, a i+r}=\sum_{i=0}^{n}\left(\frac{\lambda_{1}^{a i+r}-\lambda_{2}^{a i+r}}{\lambda_{1}-\lambda_{2}}\right) \\
& =\frac{1}{\lambda_{1}-\lambda_{2}}\left(\sum_{i=0}^{n} \lambda_{1}^{a i+r}-\sum_{i=0}^{n} \lambda_{2}^{a i+r}\right) \\
& =\frac{1}{\lambda_{1}-\lambda_{2}}\left\{\frac{\left(\lambda_{1}^{a(n+1)+\mathrm{r}}-\lambda_{1}^{r}\right)}{\lambda_{1}^{a}-1}-\frac{\left(\lambda_{2}^{a(n+1)+r}-\lambda_{2}^{r}\right)}{\lambda_{2}^{a}-1}\right\} \\
& \left(\lambda_{1} \lambda_{2}\right)^{a} \lambda_{1}^{a n+r}-\lambda_{1}^{a n+a+r}-\lambda_{1}^{r} \lambda_{2}^{a}+\lambda_{1}^{r} \\
& =\frac{-\left(\lambda_{1} \lambda_{2}\right)^{a} \lambda_{2}^{a n+r}+\lambda_{2}^{a n+a+r}+\lambda_{2}^{r} \lambda_{1}^{a}-\lambda_{2}^{r}}{\left(\lambda_{1}-\lambda_{2}\right)\left(\left(\lambda_{1} \lambda_{2}\right)^{a}-\left(\lambda_{1}^{a}+\lambda_{2}^{a}\right)+1\right)} \\
& (-2)^{a}\left(\lambda_{1}^{a n+r}-\lambda_{2}^{a n+r}\right)-\left(\lambda_{1}^{a n+a+r}-\lambda_{2}^{a n+a+r}\right) \\
& =\frac{+\left(\lambda_{1}^{r}-\lambda_{2}^{r}\right)-\left(\lambda_{1} \lambda_{2}\right)^{r}\left(\lambda_{1}^{a-r}-\lambda_{2}^{a-r}\right)}{\left(\lambda_{1}-\lambda_{2}\right)\left((-2)^{a}-\left(\lambda_{1}^{a}+\lambda_{2}^{a}\right)+1\right)} \\
& J_{k, a(n+1)+r}-(-2)^{a} J_{k, a n+r} \\
& \sum_{i=0}^{n} J_{k, a i+r}=\frac{-J_{k, r}-(-2)^{r} J_{k, a-r}}{J_{k, a+1}+2 J_{k, a-1}-(-2)^{a}-1}
\end{aligned}
$$

\subsection{Corollary 2.4}

Formula for sum of odd k-Jacobsthal numbers If $a=2 p+1$, then equation (2.6) gives

$$
\begin{gathered}
J_{k,(2 p+1)(n+1)+r} \\
-(-2)^{(2 p+1)} J_{k,(2 p+1) n+r} \\
\sum_{i=0}^{n} J_{k,(2 p+1) i+r}=\frac{-J_{k, r}-(-2)^{r} J_{k,(2 p+1)-r}}{J_{k,(2 p+2)}+2 J_{k, 2 p}} \\
-(-2)^{(2 p+1)}-1
\end{gathered}
$$

For example: (1) If $p=0$, then $a=1$ and $r=0$, we have

$$
\begin{aligned}
& \sum_{i=0}^{n} J_{k, i}=\frac{J_{k, n+1}-(-2)^{1} J_{k, n}-J_{k, 0}-(-2)^{0} J_{k, 1}}{J_{k, 2}+2 J_{k, 0}-(-2)^{1}-1} \\
& =\frac{J_{k, n+1}+2 J_{k, n}-1}{k+1}
\end{aligned}
$$

(2) If $p=1$, then $a=3$, we have

$$
\sum_{i=0}^{n} J_{k, 3 i+r}=\frac{J_{k, 3(n+1)+r}+8 J_{k, 3 n+r}-J_{k, r}-(-2)^{r} J_{k, 3-r}}{J_{k, 4}+2 J_{k, 2}+7}
$$

If $r=0$, then

$$
\begin{aligned}
& \sum_{i=0}^{n} J_{k, 3 i}=\frac{J_{k, 3(n+1)}+8 J_{k, 3 n}-J_{k, 0}-J_{k, 3}}{J_{k, 4}+2 J_{k, 2}+7} \\
& =\frac{J_{k, 3 n+3}+8 J_{k, 3 n}-\left(k^{2}+2\right)}{k^{3}+6 k+7}
\end{aligned}
$$

If $r=1$, then

$$
\begin{aligned}
& \sum_{i=0}^{n} J_{k, 3 i+1}=\frac{J_{k, 3 n+4}+8 J_{k, 3 n+1}-J_{k, 1}-(-2)^{1} J_{k, 2}}{J_{k, 4}+2 J_{k, 2}+7} \\
& =\frac{J_{k, 3 n+4}+8 J_{k, 3 n+1}-1+2 k}{k^{3}+6 k+7}
\end{aligned}
$$

If $r=2$, then

$$
\begin{aligned}
& \sum_{i=0}^{n} J_{3 i+2}=\frac{J_{k, 3 n+5}+8 J_{k, 3 n+2}-J_{k, 2}-(-2)^{2} J_{k, 1}}{J_{k, 4}+2 J_{k, 2}+7} \\
& =\frac{J_{k, 3 n+5}+8 J_{k, 3 n+2}-k-4}{k^{3}+6 k+7}
\end{aligned}
$$

(3) If $p=2$, then $a=5$, we have

$$
\begin{aligned}
& J_{k, 5(n+1)+r}-(-2)^{5} J_{k, 5 n+r} \\
\sum_{i=0}^{n} J_{k, 5 i+r}= & \frac{-J_{k, r}-(-2)^{r} J_{k, 5-r}}{J_{k, 6}+2 J_{k, 4}-(-2)^{5}-1}
\end{aligned}
$$

If $r=0$, then

$$
\sum_{i=0}^{n} J_{k, 5 i}=\frac{J_{k, 5 n+5}+32 J_{k, 5 n}-k^{4}-6 k^{2}-4}{k^{5}+10 k^{3}+20 k+31}
$$

If $r=1$, then

$$
\sum_{i=0}^{n} J_{k, 5 i+1}=\frac{J_{k, 5 n+6}+32 J_{k, 5 n+1}+2 k^{3}+4 k-1}{k^{5}+10 k^{3}+20 k+31}
$$


If $r=2$, then

$$
\sum_{i=0}^{n} J_{k, 5 i+2}=\frac{J_{k, 5 n+7}+32 J_{k, 5 n+2}-4 k^{2}-k-8}{k^{5}+10 k^{3}+20 k+31}
$$

If $r=3$, then

$$
\sum_{i=0}^{n} J_{k, 5 i+3}=\frac{J_{k, 5 n+8}+32 J_{k, 5 n+3}-k^{2}+8 k-2}{k^{5}+10 k^{3}+20 k+31}
$$

If $r=4$, then

$$
\sum_{i=0}^{n} J_{k, 5 i+4}=\frac{J_{k, 5 n+9}+32 J_{k, 5 n+4}-k^{3}-4 k-16}{k^{5}+10 k^{3}+20 k+31}
$$

\subsection{Corollary 2.5}

Sum of even k-Jacobsthal numbers.

If $a=2 p$, then equation (2.6) is

$$
\begin{gathered}
J_{k, 2 p(n+1)+r}-(4)^{p} J_{k, 2 p n+r} \\
\sum_{i=0}^{n} J_{k, 2 p i+r}=\frac{-J_{k, r}-(-2)^{r} J_{k, 2 p-r}}{J_{k, 2 p+1}+2 J_{k, 2 p-1}-(4)^{p}-1}
\end{gathered}
$$

For example: (1) If $p=1$, then $a=2$, we have

$$
\sum_{i=0}^{n} J_{k, 2 i+r}=\frac{J_{k, 2(n+1)+r}-4 J_{k, 2 n+r}-J_{k, r}-(-2)^{r} J_{k, 2-r}}{J_{k, 3}+2 J_{k, 1}-4-1}
$$

If $r=0$, then

$$
\sum_{i=0}^{n} J_{k, 2 i}=\frac{J_{k, 2(n+1)}-4 J_{k, 2 n}-k}{k^{2}-1}
$$

If $r=1$, then $\sum_{i=0}^{n} J_{k, 2 i+1}=\frac{J_{k, 2(n+1)+1}-4 J_{k, 2 n+1}+1}{k^{2}-1}$

(2) If $p=2$, then $a=4$, we have

$\sum_{i=0}^{n} J_{k, 4 i+r}=\frac{J_{k, 4(n+1)+r}-16 J_{k, 4 n+r}-J_{k, r}-(-2)^{r} J_{k, 4-r}}{J_{k, 5}+2 J_{k, 3}-17}$

If $r=0$, then

$$
\sum_{i=0}^{n} J_{k, 4 i}=\frac{J_{k, 4 n+4}-16 J_{k, 4 n}-k^{3}-4 k}{k^{4}+8 k^{2}-9}
$$

If $r=1$, then

$$
\sum_{i=0}^{n} J_{k, 4 i+1}=\frac{J_{k, 4 n+4}-16 J_{k, 4 n}+2 k^{2}+3}{k^{4}+8 k^{2}-9}
$$

If $r=2$, then

$$
\sum_{i=0}^{n} J_{k, 4 i+2}=\frac{J_{k, 4 n+6}-16 J_{k, 4 n+2}-5 k}{k^{4}+8 k^{2}-9}
$$

If $r=3$, then

$$
\sum_{i=0}^{n} J_{k, 4 i+3}=\frac{J_{k, 4 n+7}-16 J_{k, 4 n+3}+k^{2}+10}{k^{4}+8 k^{2}-9} .
$$

Now, we have considered the alternating sequence $\left\{(-1)^{n} J_{k, a n+r}\right\}$. By the previous method we can also find the sum formula for this sequence.

\subsection{Theorem 2.6}

Alternating sum of the k-Jacobsthal numbers with index $a n+r$ is given by

$$
\begin{gathered}
(-1)^{n} J_{k, a(n+1)+r} \\
+(-1)^{n}(-2)^{a} J_{k, a n+r} \\
\sum_{i=0}^{n}(-1)^{i} J_{k, a i+r}=\frac{+J_{k, r}+(-2)^{r} J_{k, a-r}}{J_{k, a}+(-2)^{a}+1}
\end{gathered}
$$

Proof: Taking L.H.S. and applying the binet formula for the k-Jacobsthal numbers equation (1.2) we have

$$
\begin{aligned}
& \sum_{i=0}^{n}=\frac{1}{\lambda_{1}-\lambda_{2}} \sum_{i=0}^{n}(-1)^{i}\left(\lambda_{1}^{a i+r}-\lambda_{2}^{a i+r}\right) \\
& =\frac{1}{\lambda_{1}-\lambda_{2}}\left\{\begin{array}{c}
\frac{\lambda_{1}^{r}\left(1+(-1)^{n} \lambda_{1}^{a(n+1)}\right)}{1+\lambda_{1}^{a}} \\
-\frac{\lambda_{2}^{r}\left(1+(-1)^{n} \lambda_{2}^{a(n+1)}\right)}{1+\lambda_{2}^{a}}
\end{array}\right\} \\
& =\frac{(-1)^{n}(-2)^{a} J_{k, a n+r}+(-1)^{n} J_{k, a(n+1)+r}}{+J_{k, r}+(-2)^{r} J_{k, a-r}} \\
& J_{k, a}+(-2)^{a}+1
\end{aligned}
$$

that is

$$
\begin{aligned}
& (-1)^{n} J_{k, a(n+1)+r}+(-1)^{n}(-2)^{a} J_{k, a n+r} \\
& \sum_{i=0}^{n}(-1)^{i} J_{k, a i+r}=\frac{+J_{k, r}+(-2)^{r} J_{k, a-r}}{J_{k, a}+(-2)^{a}+1}
\end{aligned}
$$

For different values of $a$ and $r$, the above sum can be written as

For $\quad a=1$, then $r=0, \quad$ we have

$\sum_{i=0}^{n}(-1)^{i} J_{k, 2 i}=\frac{(-1)^{n} J_{k,(n+1)}-2(-1)^{n} J_{k, n}+1}{k-1}$

For, $a=2$

$$
\sum_{i=0}^{n}(-1)^{i} J_{k, 2 i+r}=\frac{(-1)^{n} J_{k, 2(n+1)+r}+4(-1)^{n} J_{k, 2 n+r}}{+J_{k, \mathrm{r}}+(-2)^{r} J_{k, 2-r}}
$$

a) If $r=0$, then

$$
\sum_{i=0}^{n}(-1)^{i} J_{k, 2 i}=\frac{(-1)^{n} J_{k, 2 n+2}+4(-1)^{n} J_{k, 2 n}+k}{k^{2}+9}
$$

b) If $r=1$, then

$$
\sum_{i=0}^{n}(-1)^{i} J_{k, 2 i+1}=\frac{(-1)^{n} J_{k, 2 n+3}+4(-1)^{n} J_{k, 2 n+1}-1}{k^{2}+9}
$$


For $a=3$,

$$
\sum_{i=0}^{n}(-1)^{i} J_{k, 3 i+r}=\frac{(-1)^{n} J_{k, 3(n+1)+r}-8(-1)^{n} J_{k, 3 n+r}+(-2)^{r} J_{k, 3-r}}{J_{k, 3+1}+2 J_{k, 3-1}-7}
$$

If $r=0$, then

$$
\sum_{i=0}^{n}(-1)^{i} J_{k, 3 i}=\frac{(-1)^{n} J_{k, 3(n+1)}+8(-1)^{n} J_{k, 3 n}+k^{2}+2}{k^{3}+6 k-7}
$$

If $r=1$, then

$$
\sum_{i=0}^{n}(-1)^{i} J_{k, 3 i+1}=\frac{(-1)^{n} J_{k, 3 n+4}+8(-1)^{n} J_{k, 3 n+1}+1-2 k}{k^{3}+6 k-7}
$$

If $r=2$, then

$$
\sum_{i=0}^{n}(-1)^{i} J_{k, 3 i+2}=\frac{(-1)^{n} J_{k, 3 n+5}+8(-1)^{n} J_{k, 3 n+2}+k+4}{k^{3}+6 k-7}
$$

\section{Reference}

[1] Bolat C., Kose H., On the Properties of k-Fibonacci Numbers. Int. J.Contemp. Math. Sciences 2010, 22 (5), 1097-1105.

[2] Campos H., Catarino P., Aires A.P., Vasco P. and Borges A., On Some Identities of k-Jacobsthal-Lucas Numbers, Int. Journal of Math. Analysis, 2014, 8 (10), 489-494.

[3] Catarino P., On Some Identities and Generating Functions for kPell Numbers. Int. Journal of Math. Analysis 2013, 7 (38), 1877 1884.
[4] Catarino P., Vasco P., Modified k-Pell Sequence: Some Identities and Ordinary Generating Function. Applied Mathematical Sciences 2013, 7 (121), 6031-6037.

[5] Catarino P., Vasco P., On some Identities and Generating Functions for k-Pell-Lucas Sequence. Applied Mathematical Sciences 2013, 7 (98), 4867-4873.

[6] Catarino P., On Some Identities for k-Fibonacci Sequence. International Journal of Contemporary mathematical Sciences 2014, 9 (1), 37-42.

[7] Falcon S. and Plaza A., On k-Fibonacci Numbers of Arithmetic Indexes, Applied Mathematics and Computation, 2009, 208, 180185.

[8] Falcon S., On the k-Lucas Numbers, International Journal of Contemporary mathematical Sciences 2011, 6 (21), 1039-1050.

[9] Falcon S., On the k-Lucas Numbers of Arithmetic Indexes, Applied Mathematics, 2012, 3, 1202-1206.

[10] Horadam A. F., A Generalized Fibonacci Sequence, The American Mathematical Monthly, 1961, 68, 455-459.

[11] Horadam A.F., Jacobsthal Representation Numbers. Fibonacci Quarterly, 1996, 34 (1), 40-54.

[12] Jaiswal D. V., On a Generalized Fibonacci Sequence, Labdev Journal of Science and Technology, Part A, 1969, 7, 67-71.

[13] Jhala D., Sisodiya K., Rathore G.P.S., On Some Identities for kJacobsthal Numbers. Int. Journal of Math. Analysis 2013, 7 (2), 551-556.

[14] Lee G. Y., Lee S. G., Kim J. S., Shin H. K., The Binet Formula and the Representation of k-Generalized Fibonacci Numbers, Fibonacci Quarterly, 2001, 39 (2), 158-164.

[15] Lee G. Y., Lee S. G., Shin H. K., On the k-Ggeneralized Fibonacci Matrix $Q_{k}$, Linear Algebra Applications, 1997, 251, 73-88.

[16] Slone NJA, The On-Line Encyclopedia of Integer Sequences, (2006), www.research.att.com/ njas/sequences/.

[17] Walton J. E. and Horadam A. F., Some Further Identities for the Generalized Fibonacci sequence, Fibonacci Quarterly, 1974, 12 (3), 272-280.

[18] Zai L. J. and Sheng L. J., Some Properties of the Generalization of the Fibonacci Sequence, Fibonacci Quarterly, 1987, 25 (2), 111117. 